\title{
Economic Decisions on Proposed Work Environmental Studies - a Theory for Cost and Value of Information
}

\author{
Mahmoud Rezagholi \\ Department of Occupational and Public Health Sciences, University of Gävle, Gävle, Sweden \\ Email address: \\ madrei@hig.se
}

\section{To cite this article:}

Mahmoud Rezagholi. Economic Decisions on Proposed Work Environmental Studies - a Theory for Cost and Value of Information. Science Journal of Public Health. Vol. 4, No. 1, 2016, pp. 11-19. doi: 10.11648/j.sjph.20160401.12

\begin{abstract}
Assessment studies of occupational exposures are retrospectively evaluated based on their achieved statistical efficiency and/or their imposed costs. However, any decision on the performance of such studies strongly requires an economic evaluation in advance. The economic evaluation of proposed work environmental studies needs, in turn, access to information on the socio-economic impacts of occupational exposures. The present article aims to help policy makers in their decisions on proposed work environmental studies by introducing a cost-value approach to the information to be produced during the studies. The cost-value approach is not exposed to subjective judgements, as in the approach of "willingness to pay", nor to consideration of invaluable statistical efficiency as "output", as in exposure assessment studies. The work environmental study investigated in this article contained three different groups of occupational exposures that caused sickness absences and impairments at work in a Swedish company, Sandvik Materials Technology. The results show that the suggested study would be acceptable to the policy makers in the company, as its estimated value was strictly greater than its estimated costs.
\end{abstract}

Keywords: Occupational Exposures, Economics of Information, Social Benefits, Policy Makers, Economic Efficiency

\section{Introduction}

\subsection{Social Costs of Work-Related Disorders and the Progress of Exposure Assessment Studies}

Work-related disorders are known as a reason for high social costs in many countries, due to the increased sickness absences and disabilities that they produce [1-4]. The social costs are not only limited to the direct medical care expenditures; there are also indirect costs, which are estimated to be higher when increased work-related disorders result in decreased labour productivity and decreased quality of the goods and services produced $[1,3]$. Thus, many studies of occupational exposures have been designed in different countries in order to improve occupational health and avoid the huge social cost [5-8]. At the same time, the cost of these studies has also been a challenge due to the limited resources available to researchers in the field. In many cases, the sizes of studies have been cut back in terms of time and numbers of participants and exposures, in order to circumvent the economic problem. However, the economic decision of whether to perform a study, at management level, must consider the costs of work-related disorders, which can be much greater than the costs of the study. Further, constant technical developments in hardware and software continuously reduce the cost of exposure assessment studies, and make it possible to expand such studies and meet the demand for more information about occupational exposures. Thus, a holistic approach might be the dominant one in work environmental studies, when the causes of deficiencies in the body of workers include ergonomic workloads, psychosocial risk factors, chemical substances, noise, vibration, lighting, and temperature [9]. Despite technical possibilities and scientific findings, work environmental studies have generally focused on specific exposures while ignoring socio-economic impacts such as the effects on sickness absences and impairments at work, and thus on potential income loss. Hence, these studies have been concerned with the collection of data on single exposures with the least statistical error, and have therefore been evaluated in terms of their statistical efficiency [1015]. Given the scarce resources, the statistical efficiency then began to be balanced against the cost of assessing the exposure of interest, in so-called cost-efficiency studies [16, 17]. However, statistical efficiency is only one factor in 
evaluating the output of occupational exposure studies [18]. A purely efficient assessment of an exposure cannot be the basis for evaluation of the output if, for instance, the critical occupational exposures in the work environment (i. e. the exposures to disorders that are behind the social costs) are omitted in the study. By considering statistical efficiency as the actual output and not as a criterion for evaluation, resources have been allocated optimally between different stages of statistical production to approach the true average value of a single exposure variable [19-24]. Moreover, taking as an output the statistical efficiency in terms of precision and accuracy has led to obscurity in the costoutput relationship and unclear economic evaluations, as the output was not related to the social cost of work-related disorders caused by the exposure, and was also measured with different measurement units than the costs [18]. An incessant question in cost-efficiency studies is whether the higher statistical efficiency given by a more expensive design would justify the incremental cost [ibid]. Economic decision theory suggests that the cost of achieving a higher level of statistical efficiency should be compared to the opportunity cost (the expected social costs) of using the inferior study design, or with the expected social benefits of using the superior study design [ibid]. Thus, rational decision-making on investment in an exposure assessment study requires estimation of the economic outcomes with and without the use of the expensive but superior study design. In addition to this deficiency, in principle, the analysis of cost-efficiency associated with different measurement designs involves a retrospective economic evaluation; that is, an evaluation based on historical costs and known error components associated with alternative measurement designs [25-27]. However, rational decisions on the performance and expansion of exposure studies require economic evaluations in advance. Thus, the social costs of work-related disorders caused by occupational exposures cannot be ignored in economic evaluations of work environmental studies. The value of the studies' real outputs should be compared with the studies' costs and related to the social cost.

\subsection{Comprehensive View of the Work Environment}

There are multiple causes for work-related disorders which lead to sickness absences and/or impairments at work, and several risk factors in the work environment may interact and work together to create such disorders. Risk factors can be classified into three groups of exposures, concerning the psychosocial work environment, ergonomic work conditions (static and dynamic workloads), and the physical environment of the workplace. The psychosocial work environment contains risk factors such as conflicts, discrimination, stress, and low authority in execution of duties; ergonomic work conditions contain health hazards such as postural and mechanical workloads; and the physical environment contains problems with temperature, noise, lighting, air quality, and so on. Studies of occupational exposures should thus have a comprehensive view of the work environment, especially when it comes to the socioeconomic impacts of work-related disorders. Whether a decision about changes in the work environment is efficient, and its expected net benefits are great, depends on whether the information from relevant studies is complete. However, most studies in occupational health sciences are focused partially on the psychosocial work environment [5, 28, 29], the ergonomic work conditions $[6,30,31]$, and the physical characteristics of the workplace $[7,8,32]$, while the socioeconomic impacts of the work environment have not been given enough attention. One-dimensional studies of the work environment impair not only the improvement of occupational health and labour productivity, but also the estimation of the economic results and thus the valuation of such studies. Economic-oriented studies of occupational health, ranging from data collection up to intervention, cannot, at least, ignore the need for a holistic approach to the work environment and health hazards, nor can they ignore information about the effects of occupational exposures on workers' sickness absences and impairments. The economics of occupational health are based on the economic impacts of work-related disorders, which are often impossible to disentangle between the different risk factors (exposures) existing in the workplace.

\subsection{Data, Information, and Decision-Making}

Data on work-related exposures is the intermediate product to produce information about the psychological, ergonomic, and physical dimensions of the work environment. The output is produced by correcting, processing, and analysing the data and then inferring the statistical results. The partial information about each dimension of the work environment is, in turn, the input to the final output of a work environmental study that is produced by combining and transforming the informational inputs to the information used for decision-making about changing the work environment and improving occupational health. The information output, including a proposal to change the work environment, is then the input to the decision. The input-output relationships in the work environmental study can be summarized as follows:

Data on exposures $\rightarrow$ Dimensional (partial) information on the work environment $\rightarrow$ Comprehensive information on the work environment including proposals to change it $\rightarrow$ Decision.

Without further refinement and transformation to information that will be useful to the decision maker, data on occupational exposures has no value or meaning. The related data, as a set of objective facts about work-related events, is usable if it is transformed into information. Researchers transform data into useful information for decision makers in three steps: 1) correcting, processing, and analysing the data; 2) interpreting and inferring the statistical results in relation to the purpose of the work environmental study; and 3) summarizing the outcome in a concise form and developing a proposal. The information output is valued only when it is used in order to change the work environment. 


\subsection{Economics of the Information}

The first necessary step in improving occupational health is to produce useful and complete information about the work environment, and supply it to the management for a decision. When it comes to the work environment, the problem is not usually to produce information totally from scratch, but rather to produce more complete and more useful information. As the production of this information uses resources, it should initially be agreed by the management. The first economic decision is thus about producing the information. However, in making this economic decision, the cost and value of the information should be estimated and compared to each other. An economic decision is made to perform a proposed work environmental study if the expected value of the information to be produced during the study exceeds its estimated total cost. Although valuation of information improves the efficiency of this decision, it has been completely ignored in research. The term "value of information" (VOI) means that a decision which has economic consequences is made on the basis of the information provided, and so this information has an economic value [33]. Accordingly, the information output from an entire work environmental study will be used to decide whether the enclosed intervention program should be carried out in the workplace. As the total social costs caused by work-related disorders will likely be reduced by implementing the program, the information output can be valued in monetary terms both for the company and for the whole of society. The likely reduction of sickness absences and impairments at work is dependent on the content of the information, which includes a proposal to change the work environment. The expected benefits of using the information are thus a factor in determining its economic value.

The cost and value of information on the work environment can be estimated at both the micro and the macro level. The micro-economics of the information refers to the cost of producing it and the benefits of using it for individual economic units. For a rational decision on producing information at the micro level, its micro-economic value should be greater than its corresponding partial costs. The macro-economics of the information refers to its effects on the health and productivity of the national economy's labour force, gross domestic product, balance of payments, and national economic policies. The costs of production and the benefits in use of the information are estimated for the whole of the economy. The more productive the labour force, and the greater its capacity to deal with environmental problems in the workplaces, the greater the value of the information behind the relevant decisions.

Information about the work environment, as an intellectual product, is thus considered as an economic asset. However, it differs from physical assets in several ways:

1) It can be produced with minimal use of physical capital. The most useful economic resource is skilled labour (investigators and researchers).

2) Its transport costs are near to zero.
3) There is a complex relationship between its time of production and its value. Last year's information on the work environment may be worthless next year, because of changes in the underlying reality.

4) Its returns/utilities are over the long term, while all costs are paid immediately. This property may be a reason to reject applications for resource-using work environmental studies.

5) It lies somewhere between pure private goods and pure public goods, and may alternate between these poles at different stages of production and distribution. As the expected social benefits (in terms of usage and productive scheduled working hours) increase as use of the relevant information increases, there is a strong incentive to share the information across workplaces; this implies that the information counts as a public good rather than a private one. However, as the production of the information is costly for individual economic units, and the benefits fall first to them, they have an incentive to keep the information for their own use in a competitive market - "knowledge is power" [34] which gives an alternate view of it as a private good.

6) It is not consumed by being used or transmitted to other workplaces. Information on the work environment can be transmitted to all stakeholders, even during production, with no diminution of its contents.

7) As its value is not apportioned across users, it can be shared infinitely between researchers in the field and managers at relevant authorities and workplaces without losing its value. The more users for the information, the more economic benefits can be drawn from it by society when occupational health and labour productivity begin to improve. In economic terms, information on work environmental risk factors exhibits increasing returns to use.

8) The basic assumption in consumption theory that "more is better than less" is not fully met. More informative studies are valued more highly, because they lead to better decisions about the work environment. However, as decision makers have a limited capacity to process information [35], the value of information continues to increase with "more information" only up to the point at which information overload occurs.

9) Despite the cost of producing the information, it entails no real economic value without being put to use. Information about the work environment, including proposals to improve occupational health, can only carry economic value if it leads to a changed reality and results in economic benefits.

Consequently, the value of information output from work environmental studies is determined by the three following factors:

a) The maximum benefit of using the information, which is equal to the amount of disease-related costs caused by deficiencies in the work environment.

b) The probability that the associated proposal to change the work environment will be accepted by management. 
This acceptance substantially depends on the manager's budget, valuations, and preferences, as well as the price of implementing the proposal.

c) The effectiveness of the associated proposal to improve occupational health. This effectiveness is assessed via the changeability of the work environment and the content and technical characteristics of the information, which affect both the cost and the value of the information. These characteristics refer, in turn, to criteria such as relevance $^{1}$, completeness ${ }^{2}$, availability ${ }^{3}$, timeliness ${ }^{4}$, comparability ${ }^{5}$, and statistical efficiency ${ }^{6}$ associated with a work environmental study.

\subsection{Purpose and Properties}

The aim of the present article is to provide guidelines for economic decisions about proposed work environmental studies. A predictive cost-value approach to information is introduced, modelled, and then put into practice for a work environmental study intended to be proposed to the management of Sandvik Materials Technology (SMT). As the work environment is a multidimensional reality, the suggested study has a multidimensional character and is aimed at assessing exposures in the psychosocial, ergonomic, and physical work environment. Properties of the economic evaluation methodology include a productivity orientation and a societal perspective: the assessment of illness-related costs is based on sickness absenteeism and presenteeism (impairments at work) as well as low work capacity at home. In addition, all tangible illness-related costs paid by different stakeholders are defined and assessed.

\section{Methodology}

\subsection{The Suggested Work Environmental Study}

The data needed for a cost-value analysis of producing information was collected from a primary survey at SMT during May 2015, in which information about work-related sickness absenteeism and presenteeism was collected from the company's safety unit, workers, and health service. This information was then used to estimate the social costs of

1 A signal from the work environment is called information if it is relevant for decision-making; its receipt should affect the decision. Thus, even costless relevant signals are information with positive value, while costly irrelevant signals are not.

2 Completeness refers to whether the information output comprises partial information about the psychosocial, ergonomic, and physical work environment.

3 Availability refers to whether the information is available to its consumers, such as decision makers and safety units, at any time.

4 Timeliness is related to the time at which each exposure develops and the time at which the signal is received. The difference between these times (report delay) increases the uncertainty about present and future events, and thereby the expected benefits of using the information.

5 Comparability refers to comparison and connection of the outcomes with previous outcomes.

6 Statistical efficiency is concerned with the removal and reduction of statistical error. Error-exposed data lead to misinformation and then inefficient decisionmaking. The value of information increases with its statistical efficiency, though at a diminishing rate. work-related disorders [36]. In October 2015, a work environmental study involving 100 workers was suggested to SMT. This study would cover three different groups of occupational exposures (i. e. health hazards related to the psychosocial work environment, ergonomic work conditions, and the physical environment at the workplace) that cause sickness absences and impairments at work and thus create social costs. Specifically, 22 occupational health hazards would be considered: 14 psychosocial $^{7}, 3$ ergonomic $^{8}$, and 5 physical $^{9}$.

\subsection{Basic Assumptions and the Economic Model}

As the suggested work environmental study would be performed over three months, the effect of technical progress on producing the information was assumed to be constant during the study. The average variable input costs, the patterns of exposures and their impacts, the manager's behaviour, the study design, and the skills of investigators and researchers that would affect the value of the information output were assumed to be constant during the cost-value analysis. The measurement instruments and other equipment, statistical methods, and structures to display information were also assumed to be unchanged during the study.

The economic evaluation was based on the three measures described below.

a) The value of information

As the information output from the suggested work environmental study would include a proposal to prevent disorders and improve occupational health, its value would be affected by the expected social benefits of implementing the proposal. The benefits, in turn, were based on the costs of work-related disorders consisting of direct and indirect costs at the societal level. The direct costs covered the medical costs paid by different stakeholders, while the indirect costs were concerned with potential income loss.

The total social cost of disorders at SMT in 2014 (TSC) was estimated as:

$$
T S C=T M C+T P L+S I C+T R L,
$$

where $T M C$ is the total medical cost, which primarily consists of medical care expenditures such as medicines, hospitalizations, visits to doctors and other practitioners, transportation, and rehabilitation paid for by workers, employers, and the public sector; $T P L$ is the total productivity loss at both workplace and home, expressed as the number of lost working hours multiplied by the hourly median wage at national level, which is a basis to assess the marginal revenue product of labour; SIC is the cost to the Swedish Social Insurance Agency, which pays the ill workers $80 \%$ of their wage from their fifteenth sick day, to compensate for wages lost; and $T R L$ is the tax revenue loss in

7 Conflicts, insults, harassment, bullying, alienation, discrimination, low status, low salary, stress over high requirements, stress over unclear expectations, ingratitude, and problems with or lack of authority, job security, and stability. 8 Handling heavy objects, repetitiveness, and awkward body postures. 9 Problems with air quality, temperature, noise, lighting, and vibration. 
terms of the income taxes not received from the sick absent workers who get compensation less than their usual wages. Hence, in assessing total productivity loss, labour market failures (unemployment and monopsony power) and team production property (interdependence in work functions) were considered in addition to national median wages according to the human capital approach adjusted for market failure and team production (HCA-AMFTP).

Under the assumption that the increase rate in salaries for the next year would be equal to the real interest rate, the maximum social benefit expected for 2015 from using the information $(M S B)$ was estimated as:

$$
M S B=k \cdot T S C,
$$

where $k$ is the degree of disorders among workers caused by deficiencies in the work environment. The maximum social benefit would be achieved if the suggested work environmental study could supply "perfect information" and eliminate the deficiencies in practice. Thus, the value of information produced from the study (VOI) was discounted by two coefficients:

$$
V O I=\phi \cdot e \cdot M S B
$$

where $\phi$ is the probability that the information output would be used to change the work environment, estimated by asking management, safety units, and workers; and $e$ is the effectiveness rate of the associated proposal to reduce workrelated disorders and increase occupational health, assessed via the changeability of the work environment with respect to the content and characteristics of the suggested study.

\section{b) The cost of information}

The total cost of producing information (TCI) about the work environment was calculated as:

$$
T C I=T F C+100 \cdot\left(C_{S}+C_{W}+C_{E}\right)=T F C+100 \cdot\left(\bar{c}_{S} \cdot S+\bar{c}_{W} \cdot W+\bar{c}_{E} \cdot E\right),
$$

where $T F C$ is the total fixed cost of the study, consisting of costs independent of the size of the study; 100 is the number of workers intended to participate in the study; $C_{S}$ is the total cost of using an interview to produce information on a worker's psychosocial work environment; $S$ is the number of psychosocial risk factors; $\bar{c}_{S}$ is the average unit cost of producing information on a psychosocial risk factor; $C_{W}$ is the cost of using video-based observation to produce information on a worker's ergonomic work conditions; $W$ is the number of ergonomic hazards; $\bar{c}_{W}$ is the average unit cost of producing information on an ergonomic hazard; $C_{E}$ is the cost of using direct technical measurements to produce information on a worker's physical environment; $E$ is the number of physical exposures; and $\bar{c}_{E}$ is the average unit cost of producing information on a physical exposure.

Thus, $S, W$, and $E$ stand for three groups of work environmental risk factors, information on which will be combined and transformed to the information output to be used for any decision on changing the work environment. To calculate the cost of producing this information, all the input costs were estimated: the user cost of capital (equipment and buildings), the cost of maintenance and technical support of equipment (repairs, servicing, and calibration), the cost of recruiting study participants, the cost of labour for recording and analysing exposure data, the cost of labour for developing a proposal to change the work environment and improve occupational health, the cost of software used, the cost of energy and material consumed during the production, the cost of educating and training investigators, and finally the cost of controlling the quality of the collected data and the study outcomes. The user cost of capital (UCC) was estimated as the sum of the annual cost of owning and using a capital asset, equal to economic depreciation plus forgone interest. UCC included rent paid for buildings (laboratory and office spaces). The cost of training included the costs of trainers as well as salaries paid to the supervisory trainees.
The labour costs of collecting data about occupational exposures included lost working time for the participating workers.

The working life of the equipment used in the suggested study was assumed to be five years. The interest rate included a risk premium according to the capital asset pricing model (CAPM).

The inflation-adjusted user cost of capital was expressed as a rate of capital value:

$$
U C C=r \cdot P P
$$

where $P P$ is the purchasing price (i. e. the value of capital estimated in the market) and $r$ is calculated as $r=$ depreciation rate + interest rate - inflation rate.

Generally, the cost of producing information on each dimension of work environment $\left(C_{i}\right.$, where $\left.i=S, W, E\right)$ was measured by multiplying their expected input quantities and their respective unit prices as:

$$
C_{i}=K \cdot P_{K}+L \cdot P_{L}+E \cdot P_{E}+M \cdot P_{M}
$$

where $K, L, E$, and $M$ stand for capital, labour, energy, and material inputs, and $P_{K}, P_{L}, P_{E}$, and $P_{M}$ are the respective unit average prices of those inputs. Thus, the total cost associated with the work environmental study consisted of the user cost of physical capital $\left(K \cdot P_{K}\right)$ including equipment and buildings, the cost of labour $\left(L \cdot P_{L}\right)$ for collecting exposure data to produce useful information to supply to the decision-maker, and the costs of energy $\left(E \cdot P_{E}\right)$ and materials $\left(M \cdot P_{M}\right)$ consumed during the information production. The cost of energy was considered because of the inclusion of energyusing equipment in the study. The materials consumed during the study consisted of tape, batteries, electronic memory, and paper. All labour costs were estimated as a straightforward product of working hours and corresponding average wages per hour. The input prices were assumed to be as determined in 
the relevant markets during the study.

c) Economic efficiency and decision function

The value of the information output estimated by (3) was compared with the total cost of producing the output estimated by (4), for an economic evaluation of the suggested study. The suggested work environmental study should be accepted if its expected economic efficiency (ee) exceeds unity:

$$
e e\left(T S C, \bar{c}_{i}, \phi\right)=V O I / T C I>1
$$

The predictive decision function shows the economic efficiency of the suggested work environmental study as a function of the social costs of work-related disorders (TSC), the unit costs of producing informational inputs $\left(\bar{c}_{i}\right)$, and the probability of using the information produced $(\phi)$. It may also be a function of the effectiveness rate $(e)$, but only if this factor's ability to reduce the social costs has different effects on the cost and on the value of the information. The higher the social costs (and probability of using the information output), the greater the economic efficiency and the greater the probability of acceptance of the suggested study. Conversely, the higher the costs of producing informational inputs, the smaller the economic efficiency and the smaller the probability of acceptance of the study.

\section{Results}

The following tables contain detailed information about the costs, value, and economic efficiency associated with the work environmental study suggested to SMT in October 2015. The costs and the value are in SEK, rounded to the nearest hundred. As of 15 October 2015, exchange rates from EUR and USD to SEK were 9.30 and 8.12, respectively.

Table 1. Estimated costs (quotations) of producing information on the work environment: fixed costs $(F C)$ and variable costs $\left(C_{S}, C_{W}\right.$, and $\left.C_{E}\right)$.

\begin{tabular}{|c|c|c|c|c|c|}
\hline & FC & $\mathbf{C}_{\mathrm{S}}$ & $\mathbf{C}_{\mathrm{W}}$ & $\mathrm{C}_{\mathrm{E}}$ & Cost of information \\
\hline Application for ethical approval & 16000 & & & & 16000 \\
\hline Equipment and office & 40000 & & & & 40000 \\
\hline Report & 3600 & & & & 3600 \\
\hline Primary judgements of management and health service & 58500 & & & & 58500 \\
\hline Mapping and planning of study & 16200 & & & & 16200 \\
\hline Primary survey (on workers' conditions/judgements) & 27800 & & & & 27800 \\
\hline Proposal to change the work environment & 82000 & & & & 82000 \\
\hline Collection of data on psychosocial risk factors & & 1066400 & & & 1066400 \\
\hline Production of information on psychosocial risk factors & & 316300 & & & 316300 \\
\hline Collection of data on ergonomic hazards & & & 38100 & & 38100 \\
\hline Production of information on ergonomic hazards & & & 629000 & & 629000 \\
\hline Collection of data on physical exposures & & & & 457000 & 457000 \\
\hline Production of information on physical exposures & & & & 137600 & 137600 \\
\hline Total & 244100 & 1382700 & 667100 & 594600 & 2888500 \\
\hline
\end{tabular}

Table 2. Expected economic value of information and economic efficiency of the suggested work environmental study: social costs of disorders (SC); proportion of social costs of disorders that are work-related ( $k$ ); maximum social benefits of preventing work-related disorders (MSB); probability of using the information ( $\phi)$; and effectiveness rate of the proposal to prevent the work-related disorders (e). Rates are rounded to three decimal places.

\begin{tabular}{llllllll}
\hline & SC & $\mathbf{k}$ & MSB & $\phi$ & e & Value of information & Economic efficiency \\
\hline TMC & 33858700 & & & & & & \\
TPL & 1143849300 & & & & & & \\
SIA & 38802000 & & & & & & \\
TRL & 6614000 & & & & & & \\
Total & $\mathbf{1 2 2 3 1 2 4 0 0 0}$ & $\mathbf{0 . 3 6 2}$ & $\mathbf{4 4 2 8 5 5 5 0 0}$ & $\mathbf{0 . 1 8 0}$ & $\mathbf{0 . 2 7 0}$ & $\mathbf{2 1 5 2 2 8 0 0}$ & $\mathbf{7 5 1}$ \\
\hline
\end{tabular}

As shown in the tables, the cost and the value of information output that would be produced in the suggested work environmental study at SMT in 2015 were estimated according to equations (4) and (3) as 2888500 and 21522 800 SEK, respectively. The latter value is the macroeconomic value of the information, since the value is based on social benefits of preventing work-related disorders. Also, the cost of producing information is estimated at the societal level. The suggested work environmental study should thus be accepted in a social decision-making process, as its expected economic efficiency (i. e. value-cost ratio) is about 7.451 , which is strictly greater than unity.

\section{Discussion}

\subsection{Application Area of the Cost-Value Approach}

The decision problem for a proposed work environmental study can be resolved by modelling the cost of performing the study and the value of the information which would be produced during the study. To demonstrate this in a real-life case, the cost and value of the information were estimated in economic terms for a study suggested to the Swedish company Sandvik Materials Technology in 2015. However, this model has the potential to be applied, after necessary adjustments, in many 
contexts with different demands for information, and at least in the area of public health and health policy sciences. There are often reports from schools and workplaces with specific environmental deficiencies that have caused certain disorders. Suggested intervention studies dealing with the specific deficiency can easily be evaluated according to the cost-value approach to information introduced in the present article. The study of the work environment can also be evaluated according to this model when covering different companies across an industry or an occupational group in a region. Specifically, the model is particularly suited for application to macro studies of the work environment by the authorities in society. The economic decision of whether to conduct a work environmental study was the first step in attempting to improve occupational health and labour productivity. The next decision problem to be resolved concerns the associated proposal for changing the work environment. In resolving the second decision, the expected social benefits of changing the work environment $(e \cdot M S B)$ will be compared with the corresponding estimated costs.

\subsection{Technical Characteristics of Work Environmental Studies}

The technical characteristics of the information production, which referred to criteria such as statistical performance, relevance, completeness, and comparability, were assumed to have a constant effect in determining the economic value of information; that is, their effects were reflected in the proposal's rate of effectiveness in preventing work-related disorders. Thus, the size and duration of the study, the exposure measurement methods, the quality of equipment, and the level of competence and experience embodied in the investigators and researchers were assumed to be unchanged during the suggested work environmental study. The technical characteristics also had a constant effect on the cost of producing the information. The technical characteristics of measurement methods and investigators were also assumed to be homogeneous for all occupational exposures. For instance, the investigators' competences were assumed to be on the same level regardless of which dimension of the work environment they were producing information on. The measurement methods and instruments (i. e. interviews for the psychosocial risk factors, observation for the ergonomic risk factors, and direct technical measurements for the physical risk factors) were assumed to be appropriate and have the same technical performance in assessing occupational exposures. Thus, competence, appropriateness, and technical performance were assumed to be evenly distributed over the assessment of exposures in the psychosocial, ergonomic, and physical work environment.

\subsection{Cost Assessments of the Work Environmental Studies}

The reliability of the cost-value analysis of the information will, of course, be improved if more attention is paid to the construction of the model and the estimation of the input cost parameters. Ignorance of important input costs and/or wellbehaved cost models can, in turn, complicate the cost-value assessment and result in the selection of a non-optimal decision about the suggested work environmental study. The properties of the study and the specific features of the information output should be considered when identifying the fixed and variable inputs and selecting a model for calculating their costs. For instance, the total cost of the work environmental study suggested in this article included the cost of the primary survey to collect the information needed to estimate the social costs of work-related disorders. However, if this survey was performed by the company's safety unit, its cost would not need to be included in the economic evaluation of the suggested work environmental study.

Three economic aspects were considered in assessing the costs of producing information:

Macro-economics of information: Instead of partial costs for stakeholders, the social costs of using resources were considered; that is, all expenses paid for the suggested work environmental study, no matter to which part of the society the cost falls. For instance, the user costs of physical capital, the rent paid for laboratory and office spaces, costs for the primary survey, and costs for collecting data on occupational exposures can fall partially to researchers and partially to the enterprise and other stakeholders. The division between these parties was not considered in the assessment of costs. When assessing the value of information, the benefits of preventing work-related disorders were considered at the societal level, and so the cost assessment could not ignore the societal perspective.

Sunk costs: It may be the case that when performing the suggested work environmental study, some unexpected events will occur that have not been considered in the assessment of costs. These unexpected prospective costs, which cannot be recovered, are usually called "sunk costs" in economics and business decision-making. Sunk costs during work environmental studies can be a challenge when assessing the total cost of such studies. However, these costs should not be assessed at all, and were therefore not included in the cost model applied in this article. Unlike fixed costs, unexpected prospective costs should be ignored when making future economic decisions about work environmental studies. Although these costs can be incurred during a work environmental study, they cannot be recovered even if the study shuts down.

Input costs in the short run: The suggested work environmental study is intended to be performed over three months; that is, in the short run, where the inputs are divided into constant and changeable inputs, and the total cost consists of the fixed and variable costs. Fixed costs are the costs that do not vary with the magnitude of information output, and are related to the inputs that remain unchanged during the production. Fixed costs can be eliminated only by closing down the work environmental study. Variable costs are the costs that vary as output varies, and are related to the variable inputs (i. e. the numbers of workers participating in the study, and exposures in the psychological, ergonomic, and physical work environment). Thus, all fixed and variable costs matter in economic decisions about proposed work environmental studies that are usually carried out in the short run. 


\subsection{Evaluation of Methods to Estimate VOI}

Three methods can be used to assess the value of information about the work environment. The first of these is based on the historical cost to produce such information, and assumes that the value of an asset at the time of acquisition approximates the usual cost of producing it. The method is easy to use, and is widely used in practice for estimating VOI. However, historical cost may not reflect the current value, as value may vary over time [37] and be affected by the demand for information. The second method is based on the market value of information and willingness to pay (i. e. the amount of money the consumers are willing to pay for it). This method takes into account the current value of the information, but unlike the first method is purely demand-driven. The third method deals with the future economic benefits of using the information. Theoretically, this is the best approximation of the true economic value of the work environmental information. The method should also consider other important determinant factors, such as the probability that the information produced is then actually used in the workplace, as considered in the present article. The first weakness of the method is the difficulty of assessing: 1) some of the benefit variables such as human welfare (leisure, life quality, and life expectancy), and 2) the external usability of the information and the economic benefits of this. This weakness leads to underestimation of the true value of the information produced in the study. The second weakness is that the determinant factors are correlated, although this correlation does not produce an error in estimating VOI. The effectiveness of the associated proposal to change the work environment and the cost of the work-related disorders both affect the likelihood of implementing the proposal. However, the two determinant factors affect this likelihood in different directions, depending on the decision makers' valuation, expectations of future income, and budget, and the cost of realizing the proposal.

These three methods can be applied before producing the information, after producing the information but before using it, or after using the information.

Table 3. Evaluation of methods to estimate the value of information produced during work environmental studies, ranked according to their appropriateness and efficiency at three time points.

\begin{tabular}{|c|c|c|c|}
\hline Value of information & Historical cost & Willingness to pay & Future economic benefits \\
\hline Before production & Good & Better & Best \\
\hline After production; before using & Better & Good & Best \\
\hline After using & Good & Better & Best \\
\hline
\end{tabular}

Until now, none of these methods has been put into practice to assess the economic value of information produced during work environmental studies. The model for estimating VOI introduced in the present article is the most appropriate and efficient in predictive economic decision-making. However, the value obtained from the model approaches the true economic value when the model is used after production, because the determinant factors $\phi$ and $e$ can be assessed more efficiently. It approaches the true economic value even more closely after using the information, when both the economic benefits and $e$ are known.

\subsection{Economic Efficiency of Work Environmental Studies}

The features and properties of work environmental studies (i. e. characteristics of study design, measurement methods, equipment, and investigators) have very different effects on the cost and value of the information output. The net value of the information output would thus vary as investment in the inputs varies. Assuming a changeable work environment and a linear property, in addition to a promised use of the information output, the economic efficiency of the proposed study would simplify to a cost ratio: the costs of work-related disorders divided by the cost of the study.

\section{Conclusion}

It is suggested that the work environmental study proposed to SMT should be evaluated in advance based on the costvalue approach of the information that would be produced during the study. The study would encompass critical exposures in the psychosocial, ergonomic, and physical work environment, and include a proposal to change the work environment and improve occupational health. The economic cost of producing the information was estimated to be 2888 500 SEK, while the economic value of the information was 21522800 SEK. As the economic efficiency or value-cost ratio of the suggested work environmental study was estimated to be 7.451, much larger than unity, the performance of the study should be accepted in a rational social decision-making process.

\section{Acknowledgements}

The Faculty of Health and Occupational Studies from University of Gävle-Sweden and the Swedish company Sandvik Materials Technology are gratefully acknowledged for their support and cooperation in writing this article.

\section{References}

[1] Safe Work Australia. The cost of work-related injury and illness for Australian employers, workers and the community: 2008-09. 2012 ISBN 978-0-642-33381-0.

[2] Punnett L, Wegman DH. Work-related musculoskeletal disorders: the epidemiologic evidence and the debate. J Electromyogr Kinesiol 2004; 14: 13-23.

[3] Piedrahita H. Costs of work-related musculoskeletal disorders (MSDs) in developing countries: Colombia case. JOSE 2006; 12: 379-386. 
[4] Lubeck DP. The costs of musculoskeletal disorders: Health needs assessment and health economics. Best practice \& Research 2003; 17: 529-539.

[5] Smith PM, Glazier RH, Lu, Mustard CA. The psychosocial work environment and incident diabetes in Ontario, Canada. Occupational Medicine 2012; 62: 413-419.

[6] Paudyal P, Ayres JG, Semple S, Macfarlane GJ. Low back pain among textile workers: a cross-sectional study. Occupational Medicine 2013; 63: 129-134.

[7] Tan HH, Teo S, Tseng HC. Work-related chemical exposures presenting to an emergency department in Singapore. Occupational Medicine 2014; 64: 113-119.

[8] Xiang J, Bi P, Pisaniello D, Hansen A, Sullivan T. Association between high temperature and work-related injuries in Adelaide, South Australia, 2001-2010. Occup Environ Med 2014; 71: 246-252.

[9] David GC. Ergonomic methods for assessing exposure to risk factors for work-related musculoskeletal disorders. Occup Med 2005; 55: 190-199.

[10] Ignacio JS, Bullock WH. A strategy for assessing and managing occupational exposures. AIHA Press 2006; ISBN 1931504-69-5.

[11] Van der Beek AJ, Mathiassen SE, Burdorf A. Efficient assessment of exposure to manual lifting using company data. Applied Ergonomics 2013; 44 (3): 360-365.

[12] Warheit DB, Borm PJA, Hennes C, Lademann J. Testing strategies to establish the safety of nanomaterials: conclusions of an ECETOC workshop. Inhalation Toxicology 2007; 19: 631-643.

[13] Bourgkard E, Wild P, Gonzalez M, Févotte J, Penven E, Paris C. Comparison of exposure assessment methods in a lung cancer case-control study: performance of a lifelong taskbased questionnaire for asbestos and PAHs. Occup Environ Med 2013; 70 (12): 884-891.

[14] Takala EP, Pehkonen I, Forsman M et al. Systematic evaluation of observational methods assessing biomechanical exposures at work. Scand J Work Environ Health 2010; 36: 3-24.

[15] Loomis D and Kromhout H. Exposure variability: concepts and applications in occupational epidemiology. Am J Ind Med 2004; 45: 113-122.

[16] Rezagholi M, Mathiassen SE. Cost-efficient design of occupational exposure assessment strategies - a review. Ann Occup Hyg 2010; 54: 858-868.

[17] Rezagholi M, Mathiassen SE, Liv P. Cost Efficiency Comparison of Four Video-based Techniques for Assessing Upper Arm Postures. Ergonomics 2012; 55:350-360.

[18] Rezagholi M. Cost-Efficient Designs for Assessing WorkRelated Biomechanical Exposures. Doctoral thesis; Uppsala University 2012; p 52-53, 55-57, and 57-59.

[19] Rezagholi M, Bantekas A. Optimizing the Fraction of Expensive Direct Measurements in an Exposure Assessment Study. International Journal of Statistics in Medical Research 2014; 3: 44-45.

[20] Rezagholi M. Deriving Cost-Efficient Strategies for Observational Assessments of Postural Loads. Occup Med Health Aff 2014; 2; 4.
[21] Armstrong B (1996) Optimizing power in allocating resources to exposure assessment in an epidemiologic study. Am J Epidemiol; 144: 192-197.

[22] Duan N, Mage DT. (1997) Combination of direct and indirect approaches for exposure assessment. J Exp Anal Environ Epidemiol; 7: 439-470.

[23] Stram DO, Longnecker MP, Shames L, Kolonel LN, Wilkens LR, Pike MC, Henderson BE. (1995) Cost-efficient design of a diet validation study. Am J Epidemiol; 142: 353-362.

[24] Whitmore RW, Pellizzari ED, Zelon HS, et al. (2005) Cost/variance optimization for human exposure assessment studies. J exposure analysis environ epidemiol; 15: 464-472.

[25] Cochran WG. Sampling techniques. Wiley 1997; ISBN 0-47116240-X.

[26] Groves RM. Survey errors and survey costs. Wiley 2004; ISBN 978-0-471-67851-9.

[27] Sukhatme PV, Sukhatme BV, Sukhatme S, Asok C. Sampling theory of surveys with applications. Iowa State University Press, Ames, IA 1984.

[28] Reineholm C. Psychosocial work conditions and aspects of health. Linköping University Medical Dissertations 2013; No. 1366.

[29] Lohela M, Björklund C, Hagberg J, Vingård E, Jensen I. Does a change in psychosocial work factors lead to a change in employee health? J Occup Environ Med 2009; 51: 195203.

[30] Yazdani A, Neumann P, Imbeau D, Bigelow P, Pagell M, Theberge N, Hilbrecht M, Wells R. How compatible are participatory ergonomics programs with occupational health and safety management systems? Scand J Work Environ Health 2015; 41(2): 111-123.

[31] Cantley LF, Taiwo OA, Galusha D, Barbour R, Slade MD, Tessier-Sherman B, Cullen MR. Effect of systematic ergonomic hazard identification and control implementation on musculoskeletal disorder and injury risk. Scand J Work Environ Health 2014; 40(1): 57-65.

[32] Yogeswara T, Siddiqui NA, Hamsagar RS, Muenster RN. Industrial noise pollution and its effects on hearing capacities of workers: a research study of noise reduction from long product mill. J Industrial Pollution Control 2013; 29 (2): 185191.

[33] Feltham GA. The value of information. The Accounting Review 1968; 43: 684-696.

[34] Davenport T. H. Saving IT's soul: human centred information management. Harvard Business Review 1994.

[35] Newell A, Simon H. A. Human problem solving. PrenticeHall 1972.

[36] Rezagholi M, Bantekas A. Making economic decisions for improving occupational health - a predictive cost-benefit analysis. Occup Med Health Aff 2015; 3:6.

[37] Ijiri Y. A. A defence for historical cost accounting in R. R. Sterling (ed.): asset valuation and income determination. Scholars Book Co 1971; 1-14. 